\title{
Entropia: Um Novo Método de Mensuração da Profundidade da Anestesia. Estudo Comparativo com o Índice Bispectral na Avaliação Clínica da Intubação Traqueal com Sevoflurano *
}

\section{Entropy: A New Method of Measuring Depth of Anesthesia. Comparative Study with Bispectral Index during Clinical Evaluation in Tracheal Intubation of Patients Anesthetized with Sevoflurane}

Rogean Rodrigues Nunes, TSA ${ }^{1}$

\section{RESUMO}

Nunes RR - Entropia: Um Novo Método de Mensuração da Profundidade da Anestesia. Estudo Comparativo com o Índice Bispectral na Avaliação Clínica da Intubação Traqueal com Sevoflurano

JUSTIFICATIVA E OBJETIVOS: Entropia espectral, um novo método de análise do EEG, baseado na quantificação do caos do EEG, foi desenvolvido para monitorização da profundidade anestésica. Ele separa a monitorização em dois tipos de análise: entropia de estado $(S E)$, que inclui sinais de baixa freqüência $(<32 \mathrm{~Hz})$ e entropia de resposta $(R E)$, que inclui sinais com freqüência até $47 \mathrm{~Hz}$. O objetivo deste estudo foi comparar os valores de entropia com os do BIS e respostas sub-corticais à intubação orotraqueal, em pacientes submetidos à anestesia geral com sevoflurano.

MÉTODO: Participaram do estudo 36 pacientes com idades entre $20 \mathrm{e}$ 44 anos, ASA I, distribuídos em quatro grupos de nove, submetidos à intubação orotraqueal (IOT). Em todos os grupos, a anestesia foi induzida com sevoflurano, associado ou não ao fentanil, de acordo com o seguinte: $\left(G 1\right.$ = sevoflurano e $2,5 \mu \mathrm{g} \cdot \mathrm{kg}^{-1}$ de fentanil; $\mathrm{G} 2=$ sevoflurano e $5 \mu \mathrm{g} \cdot \mathrm{kg}^{-1}$ de fentanil; G3 = sevoflurano e $7,5 \mu \mathrm{g} \cdot \mathrm{kg}^{-1} \mathrm{de}$ fentanil e G4 = sevoflurano e solução fisiológica). Foram avaliados os seguintes parâmetros: PAS, PAD, FC, BIS, SE, RE, concentração expirada do sevoflurano (CE) e resposta motora à $I O T$ em três momentos: $M_{1}=$ imediatamente antes da indução; $M_{2}=$ imediatamente antes da intubação traqueal e $M_{3}=u m$ minuto após a intubação traqueal.

RESULTADOS: Os valores de BIS e SE variaram de maneira linear em todos os grupos, com diferenças significativas entre $M_{2}$ e $M_{3}$ nos grupos $G 1$ e G4, tendo ambos (BIS e SE) apresentado valores acima dos limitrofes entre consciência e inconsciência no momento $M_{3}$ do G4. Em relação ao RE, apenas o $G 3$ não mostrou variações estatisticamente significativas entre os momentos $M_{2}$ e $M_{3}$. As variações hemodinâmicas não ultrapassaram valores clinicamente significativos, exceto elevações da FC no G4 entre os momentos $M_{1}$ e

* Recebido do (Received from) Serviço de Anestesiologia do Hospital São Lucas de Cirurgia \& Anestesia, Fortaleza, CE; Trabalho vencedor do Prêmio Carlos Parsloe de 2003 em Anestesia Inalatória

1. Diretor Clínico e Chefe do Serviço de Anestesiologia do Hospital São Lucas - de Cirurgia \& Anestesia; Mestre em Cirurgia - Área de Concentração: Anestesiologia pela UFC; Membro da Sociedade Brasileira de Engenharia Biomédica; Graduando em Engenharia Eletrônica pela Universidade de Fortaleza

Apresentado (Submitted) em 30 de junho de 2003

Aceito (Accepted) para publicação em 07 de outubro de 2003

Endereço para correspondência (Correspondence to)

Dr. Rogean Rodrigues Nunes

Rua Gothardo Moraes, 155/1201 Bloco Dunas Papicu

60190-801 Fortaleza, CE

E-mail: rogean@fortalnet.com.br

๑) Sociedade Brasileira de Anestesiologia, 2004
$M_{3}(p<0,05 \%)$. No $G 1,66 \%$ dos pacientes reagiram as manobras de IOT e $100 \%$ no grupo $G 4$.

CONCLUSÕES: Este estudo indica que o sevoflurano isoladamente, associado a 2,5 $\mu \mathrm{g} \cdot \mathrm{kg}^{-1}$ ou $5 \mu \mathrm{g} \cdot \mathrm{kg}^{-1}$ de fentanil, não bloqueia efetivamente as respostas dos componentes cortical e sub-cortical do SNC, sendo a dose de $7,5 \mu \mathrm{g} \cdot \mathrm{kg}^{-1}$ a melhor associação ao sevoflurano para controle destes componentes anestésicos.

Unitermos: ANALGÉSICOS, Opióides: fentanil; ANESTÉSICOS, Volátil: sevoflurano; MONITORIZAÇÃO, índice bispectral, entropia de estado, entropia de resposta

\section{SUMMARY}

Nunes RR - Entropy: ANew Method of Measuring Depth of Anesthesia. Comparative Study with Bispectral Index during Clinical Evaluation in Tracheal Intubation of Patients Anesthetized with Sevoflurane

BACKGROUND AND OBJECTIVES: Spectral entropy, a new EEG analysis method based on the quantification of EEG chaos, was developed to monitor anesthetic depth. The spectral entropy involves two distinct types of analysis: state entropy (SE), which includes low frequency signals $(<32 \mathrm{~Hz})$, and response entropy $(R E)$, which includes signals up to $47 \mathrm{~Hz}$. This study aimed at comparing entropy-derived values to BIS-derived values and sub-cortical (autonomic and somatic) responses recorded during tracheal intubation in patients submitted to general anesthesia with sevoflurane.

METHODS: Participated in this study 36 patients ASA I, aged 20 to 44 years, assigned to four groups (G1-G4) of nine patients submitted to tracheal intubation (TI). In all groups anesthesia was induced with sevoflurane, associated or not to fentanyl, according to the following regimens: $G 1$ = sevoflurane plus $2.5 \mu \mathrm{g} . \mathrm{kg}^{-1}$ fentanyl; G2 = sevoflurane plus $5 \mu \mathrm{g} . \mathrm{kg}^{-1}$ fentanyl; G3 = sevoflurane plus $7.5 \mu \mathrm{g} . \mathrm{kg}^{-1}$ fentanyl; and G4 = sevoflurane plus saline solution. The following parameters were monitored: $S B P, D B P, H R, B I S, S E, R E$, sevoflurane expired concentration $(E C)$ and motor response to $T I$ at three moments: $M_{1}=$ immediately before induction; $M_{2}=$ immediately before tracheal intubation and $M_{3}=$ one minute after tracheal intubation.

RESULTS: BIS and SE values have linearly varied in all groups, with significant differences between $M_{2}$ and $M_{3}$ for Groups 1 and 4. At $M_{3}$, $B I S$ and SE values in G4 were above those for the threshold between consciousness and unconsciousness. Hemodynamic changes were not clinically significant, with the exception of HR increase between $M_{1}$ and $M_{3}$ for $G 4(p<0.05 \%)$. In $G 1,66 \%$ of patients have reacted to $\mathrm{TI}$ maneuvers as compared to $100 \%$ in G4.

CONCLUSIONS: Our findings suggest that sevoflurane alone or in association with $2.5 \mu \mathrm{g} . \mathrm{kg}^{-1}$ or $5 \mu \mathrm{g} . \mathrm{kg}^{-1}$ fentanyl does not effectively block CNS cortical and subcortical components responses during tracheal intubation, being sevoflurane plus $7.5 \mu \mathrm{g} . \mathrm{kg}^{-1}$ fentanyl the best association to control anesthetic components.

Key Words: ANALGESICS, Opioids: fentanyl; ANESTHETICS, Volatile: sevoflurane; MONITORING, bispectral index, state entropy, response entropy 


\section{INTRODUÇÃO}

A entropia espectral, um novo método de análise do EEG, baseada na quantificação do caos do EEG, foi desenvolvida para monitorização da profundidade anestésica ${ }^{1,2}$. Assim como no BIS, uma escala de 0 a 100 também é utilizada. Além disso, ele separa a monitorização em dois tipos de respostas: entropia de estado, que inclui sinais com freqüências até $32 \mathrm{~Hz}$ (quantifica a atividade cortical cerebral hipnose) e a entropia de resposta que inclui sinais com freqüências até $47 \mathrm{~Hz}$ (avalia atividade eletromiográfica de superfície-componente sub-cortical).

O objetivo deste estudo foi comparar os valores de entropia, tanto de estado como de resposta, com os do BIS na avaliação das respostas cortical e sub-cortical (autonômicas e somáticas) durante intubação orotraqueal (IOT), em pacientes submetidos à anestesia geral com sevoflurano associado ou não ao fentanil.

\section{MÉTODO}

Após aprovação pelo Comitê de Ética da instituição, participaram do estudo, aleatório e duplamente encoberto, 36 pacientes, com idades entre 20 e 44 anos, de ambos os sexos, estado físico ASA I, Mallampati classe I, extensão da cabeça normal, índice biomassa entre 21 e 28 . Foram excluídos do protocolo os pacientes em uso de medicamentos que reconhecidamente afetassem o eletroencefalograma, a CAM do sevoflurano, usos de drogas ilícitas, uso de medicamentos sedativos nas últimas 48 horas ou doenças neuromusculares. Todos os pacientes foram induzidos com sevoflurano e distribuídos em quatro grupos (G1, G2, G3 e G4, de acordo com a dose de opióide (fentanil) injetada. Os grupos foram constituídos de oito pacientes cada. A indução da anestesia foi feita com sevoflurano pela técnica do volume corrente, com demanda inicial percentual do vaporizador estabelecida em $5 \%$ e fluxo de $\mathrm{O}_{2}$ igual a $4 \mathrm{I}$. $\mathrm{min}^{-1}$, até atingir um valor de índice bispectral de $65^{3}$ (limite entre consciência e inconsciência), momento em que foi injetado fentanil nas seguintes doses: Grupo G1 (2,5 $\left.\mu \mathrm{g} . \mathrm{kg}^{-1}\right)$; Grupo G2 (5 $\left.\mu \mathrm{g} . \mathrm{kg}^{-1}\right)$; Grupo G3 $\left(7,5 \mu \mathrm{g} \cdot \mathrm{kg}^{-1}\right)$ e Grupo G4 solução fisiológica sendo aguardados cinco minutos para que se procedesse a intubação orotraqueal, a qual foi realizada pelo mesmo anestesiologista e utilizando apenas um tipo de lâmina (Macintosh $)^{4}$, com a concentração expirada de anestésico (sevoflurano) ajustada, neste momento, para manter um BIS de 30 (estabilizado em 5 minutos), com ventilação manual sob máscara.

Foram avaliados três momentos sucessivos em cada grupo $\left(M_{1}=\right.$ imediatamente antes da indução anestésica; $M_{2}=$ imediatamente antes da intubação orotraqueal e $\mathrm{M}_{3}=$ um minuto após a intubação orotraqueal) observando-se os seguintes parâmetros: índice bispectral (BIS), entropia de estado (SE), entropia de resposta (RE), concentração expirada do sevoflurano (CE), pressão arterial sistólica (PAS), pressão arteri- al diastólica (PAD) e freqüência cardíaca (FC). Foi anotada, ainda, a resposta muscular motora esquelética como parâmetro de profundidade anestésica adequada ${ }^{5}$ durante a intubação orotraqueal. Em relação aos parâmetros hemodinâmicos, foram considerados como limites inferiores clinicamente aceitáveis, PAS: $75 \mathrm{mmHg}$ e PAD: $55 \mathrm{mmHg}$. Os limites superiores foram considerados como clinicamente importantes acima de $30 \%$ dos valores de $\mathrm{M}_{1}$. Em relação à $\mathrm{FC}$, variações maiores que $30 \%$ em relação ao observado em $M_{1}$, foram também considerados de importância clínica. Em relação aos valores das entropias, a entropia de estado varia de 0 a 91 (durante anestesia adequada entre 40 e 60) e a entropia de resposta (varia de 0 a 100 - com valores adequados entre 40 e 60) sendo seus valores iguais ou maiores que a SE, quando houver ativação eletromiográfica. Esta ativação é vista como um "gap" entre RE e SE ${ }^{6}$, o qual deve ser mantido abaixo de 10. Os eletrodos foram colocados nos pacientes (BIS e entropia) nas regiões fronto-temporais de tal modo que o BIS avaliasse o canal esquerdo e a entropia o canal direito, sendo a mensuração inicial anotada após solicitar que o paciente permanecesse com os olhos fechados. Em nenhum dos pacientes foi utilizada medicação pré-anestésica.

Os resultados foram submetidos a tratamento estatístico através da Análise de Variância sendo aplicado o teste de Tukey entre níveis de fatores envolvidos, cujo valor de $p<0,05$ foi considerado estatisticamente significativo.

\section{RESULTADOS}

Os dados demográficos estão listados na tabela I.

Tabela I - Dados Demográficos (Média \pm DP)

\begin{tabular}{lcccc}
\hline Variáveis & \multicolumn{4}{c}{ Grupos } \\
\hline & $\begin{array}{c}\text { Fentanil } \\
(2 \mu \mathrm{g})\end{array}$ & $\begin{array}{c}\text { Fentanil } \\
(5 \mu \mathrm{g})\end{array}$ & $\begin{array}{c}\text { Fentanil } \\
7,5 \mu \mathrm{g})\end{array}$ & $\begin{array}{c}\text { Solução } \\
\text { Fisiológica }\end{array}$ \\
\hline Peso (kg & $56,64 \pm 2,61$ & $58,80 \pm 3,17$ & $61,00 \pm 4,67$ & $49,55 \pm 5,14$ \\
Idade (anos) & $27,30 \pm 7,10$ & $22,34 \pm 5,44$ & $27,99 \pm 6,90$ & $24,32 \pm 4,12$ \\
Altura $(\mathrm{m})$ & $1,61 \pm 0,05$ & $1,56 \pm 0,09$ & $1,53 \pm 0,06$ & $1,58 \pm 0,12$ \\
\hline
\end{tabular}

Os valores médios da PAS, PAD e FC, nos três momentos estudados, podem ser vistos na tabela II e figuras 1, 2 e 3. Por essa tabela, observa-se que houve redução estatisticamente significante $(p<0,05)$ apenas nos momentos $M_{1}$ e $M_{3}$, nos grupos G1, G2 e G3. No Grupo G4, não houve variação estatisticamente significante em relação a PAS. Para a variável $P A D$, comparando-se $M_{1}$ e $M_{3}$, houve diferença significante apenas nos grupos $\mathrm{G} 1$ e G4 ( $p<0,05 \%$ ), o que não foi observado para os grupos $\mathrm{G} 2$ e G3. Para a FC, comparando-se $\mathrm{M}_{1}$ e $\mathrm{M}_{3}$, houve diferenças significativas entre todos os grupos. Do ponto de vista clínico, contudo, de acordo com critérios pré-fixados, as variações observadas não foram importantes, exceto a elevação de FC no G4. No G1 houve reação 
motora à IOT em $66 \%$ dos casos. No G4, todos os pacientes apresentaram reação motora às manobras de intubação. Os valores médios de BIS, SE e RE podem servistos na tabela III e figuras 4, 5 e 6 . Por essa tabela e figuras observa-se que o BIS não apresentou modificação estatisticamente significante nos grupos $\mathrm{G} 2$ e G3, entre os momentos $\mathrm{M}_{2}$ e $\mathrm{M}_{3}$, sendo observado aumento estatisticamente significante do BIS entre $M_{2}$ e $M_{3}$ para os grupos G1 e G4 ( $p<0,05 \%$ ), atingindo valores limítrofes entre consciência e inconsciência (acima de 68) (Figura 4).

ASE não mostrou variação estatisticamente significante entre $\mathrm{M}_{2}$ e $\mathrm{M}_{3}$ comparando-se os grupos $\mathrm{G} 2$ e G3, sendo observado diferenças significativas entre os momentos $M_{2}$ e $M_{3}$ para os grupos G1 e G4 (variações paralelas ao BIS).

Para os valores de RE, contudo, ocorreram aumentos estatisticamente significantes entre os momentos $M_{1}$ e $M_{3}$ para os grupos G1, G2 e G4, excetuando-se apenas o G3, onde entre $\mathrm{M}_{2}$ e $\mathrm{M}_{3}$ op-valor foi > 0,05 e "gap" SE-RE menor que 10.

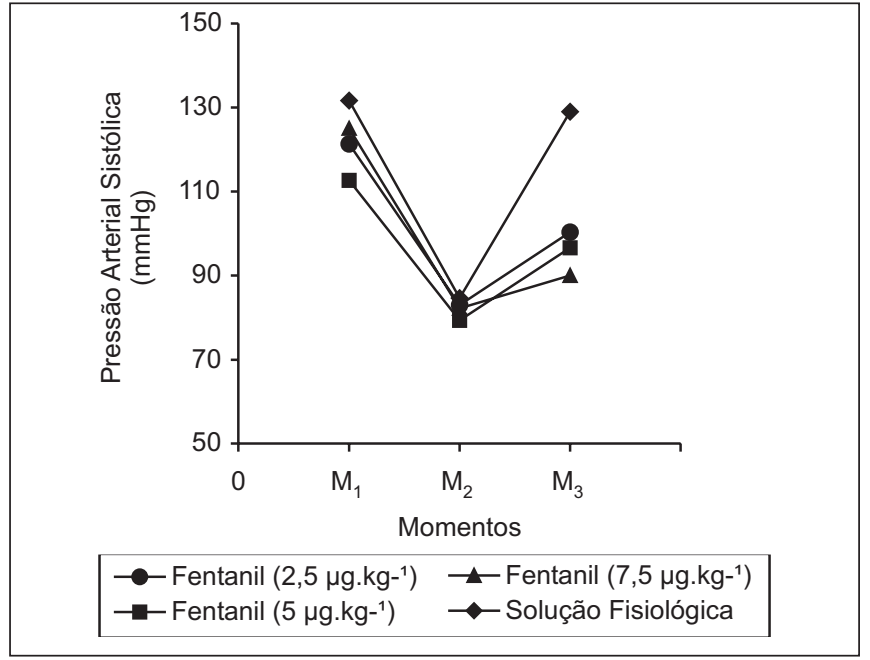

Figura 1 - Variação da Pressão Arterial Sistólica $(\mathrm{mmHg})$ $\left(M_{1} \neq M_{3}\right.$ em G1, G2 e G3 - $\left.p<0,05 . M_{1}=M_{3}\right)$. em G4 - $\left.p>0,05\right)$ Análise estatística pelo teste de Tukey

Tabela II - Pressão Arterial Sistólica, Pressão Arterial Diastólica e Freqüência Cardíaca nos Quatro Grupos e nos Momentos Estudados (Média \pm DP)

\begin{tabular}{|c|c|c|c|c|c|}
\hline \multirow[t]{2}{*}{ Momentos } & \multirow[t]{2}{*}{ Variáveis } & \multicolumn{4}{|c|}{ Grupos } \\
\hline & & $\begin{array}{c}\text { G1 = Fentanil } \\
\left(2,5 \mu \mathrm{g} \cdot \mathrm{kg}^{-1}\right)\end{array}$ & $\begin{array}{c}\mathrm{G} 2=\text { Fentanil } \\
\left(5 \mu \mathrm{g} \cdot \mathrm{kg}^{-1}\right)\end{array}$ & $\begin{array}{c}\text { G3 = Fentanil } \\
\left(7,5 \mu \mathrm{g} \cdot \mathrm{kg}^{-1}\right)\end{array}$ & $\begin{array}{c}\text { G4 = Solução } \\
\text { Fisiológica }\end{array}$ \\
\hline \multirow[t]{3}{*}{$\mathrm{M}_{1}$} & PAS & $121,30 \pm 13,90$ & $112,67 \pm 10,07$ & $125,00 \pm 6,56$ & $131,67 \pm 9,04$ \\
\hline & PAD & $81,00 \pm 8,44$ & $71,00 \pm 2,00$ & $68,33 \pm 4,60$ & $64,31 \pm 6,63$ \\
\hline & $\mathrm{FC}$ & $71,30 \pm 10,69$ & $75,00 \pm 15,70$ & $79,32 \pm 6,50$ & $83,00 \pm 8,70$ \\
\hline \multirow[t]{3}{*}{$\mathrm{M}_{2}$} & PAS & $83,00 \pm 10,23$ & $79,35 \pm 4,15$ & $82,20 \pm 5,19$ & $84,65 \pm 4,44$ \\
\hline & PAD & $56,00 \pm 4,75$ & $57,67 \pm 3,32$ & $54,00 \pm 6,00$ & $53,66 \pm 6,12$ \\
\hline & $\mathrm{FC}$ & $68,00 \pm 9,17$ & $57,00 \pm 3,00$ & $62,30 \pm 1,53$ & $80,60 \pm 14,15$ \\
\hline \multirow[t]{3}{*}{$\mathrm{M}_{3}$} & PAS & $100,33 \pm 6,01$ & $96,60 \pm 4,31$ & $90,00 \pm 10,58$ & $129,00 \pm 12,06$ \\
\hline & PAD & $79,00 \pm 9,52$ & $63,33 \pm 10,53$ & $60,00 \pm 10,49$ & $82,67 \pm 12,62$ \\
\hline & $\mathrm{FC}$ & $78,33 \pm 10,20$ & $69,44 \pm 9,18$ & $65,60 \pm 4,60$ & $117,00 \pm 10,14$ \\
\hline
\end{tabular}

$\mathrm{PAS}=\mathrm{mmHg}, \mathrm{PAD}=\mathrm{mmHg}, \mathrm{FC}=\mathrm{bpm}$

Tabela III - BIS, SE, RE e CE nos Quatro Grupos e nos Momentos Estudados (Média \pm DP)

\begin{tabular}{|c|c|c|c|c|c|}
\hline \multirow[t]{2}{*}{ Momentos } & \multirow[t]{2}{*}{ Variáveis } & \multicolumn{4}{|c|}{ Grupos } \\
\hline & & $\begin{array}{c}\text { G1 = Fentanil } \\
\left(2,5 \mu \mathrm{\mu g} \cdot \mathrm{kg}^{-1}\right)\end{array}$ & $\begin{array}{c}\mathrm{G} 2=\text { Fentanil } \\
\left(5 \mu \mathrm{kg} \cdot \mathrm{kg}^{-1}\right)\end{array}$ & $\begin{array}{l}\text { G3 = Fentanil } \\
(7,5 \mu \mathrm{g} \cdot \mathrm{kg}-1)\end{array}$ & $\begin{array}{c}\mathrm{G} 4=\text { Solução } \\
\text { Fisiológica }\end{array}$ \\
\hline \multirow[t]{4}{*}{$\mathrm{M}_{1}$} & BIS & $96,33 \pm \pm 2,00$ & $95,30 \pm 1,43$ & $93,68 \pm 1,58$ & $94,67 \pm 3,00$ \\
\hline & SE & $89,67 \pm 2,28$ & $90,00 \pm 1,25$ & $88,00 \pm 2,58$ & $89,00 \pm 1,61$ \\
\hline & RE & $95,40 \pm 3,10$ & $94,00 \pm 3,42$ & $96,67 \pm 0,84$ & $94,32 \pm 3,6$ \\
\hline & $\mathrm{CE}$ & $0,00 \pm 0,00$ & $0,00 \pm 0,00$ & $0,00 \pm 0,00$ & $0,00 \pm 0,00$ \\
\hline \multirow[t]{4}{*}{$\mathrm{M}_{2}$} & BIS & $30,00 \pm 0,00$ & $30,00 \pm 0,00$ & $30,00 \pm 0,00$ & $30,00 \pm 0,00$ \\
\hline & SE & $32,67 \pm 3,00$ & $33,33 \pm 3,81$ & $33,87 \pm 2,69$ & $36,33 \pm 2,60$ \\
\hline & $\mathrm{RE}$ & $39,07 \pm 1,46$ & $39,27 \pm 2,20$ & $35,90 \pm 1,70$ & $40,67 \pm 1,20$ \\
\hline & CE & $3,83 \pm 0,53$ & $3,57 \pm 0,40$ & $4,40 \pm 0,54$ & $4,62 \pm 0,61$ \\
\hline \multirow[t]{4}{*}{$\mathrm{M}_{3}$} & BIS & $44,33 \pm 2,18$ & $34,33 \pm 3,60$ & $36,00 \pm 4,50$ & $68,00 \pm 5,6$ \\
\hline & SE & $46,33 \pm 2,75$ & $35,32 \pm 4,51$ & $37,30 \pm 2,81$ & $66,66 \pm 3,02$ \\
\hline & RE & $65,27 \pm 2,16$ & $55,50 \pm 2,41$ & $40,21 \pm 2,80$ & $79,34 \pm 4,57$ \\
\hline & CE & $3,60 \pm 0,30$ & $3,50 \pm 0,45$ & $3,30 \pm 0,21$ & $4,40 \pm 0,75$ \\
\hline
\end{tabular}




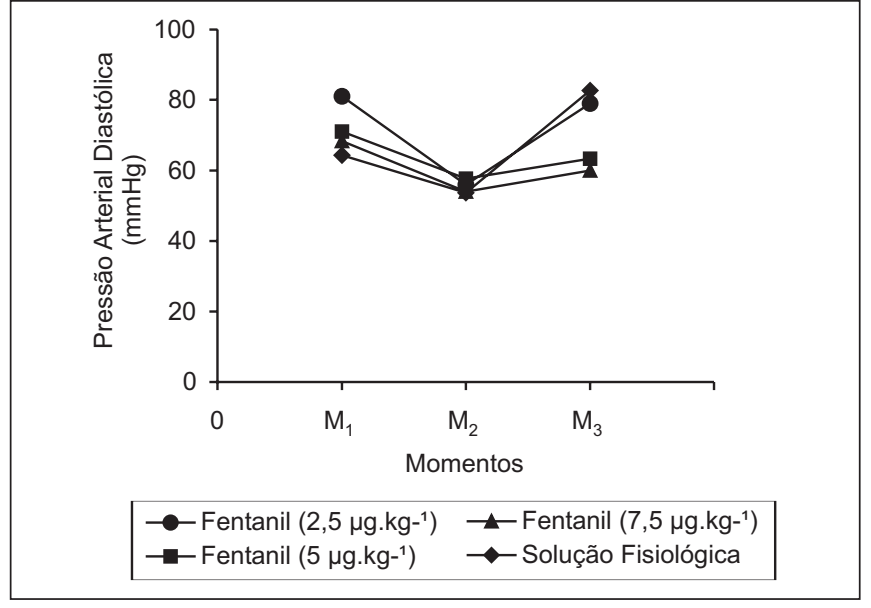

Figura 2 - Variação da Pressão Arterial Diastólica $(\mathrm{mmHg})$

$\left(M_{1} \neq M_{3}\right.$ em G1 e G4 - $p<0,05 . M_{1}=M_{3}$ em G2 e G3 - $\left.p>0,05\right)$

Análise estatística pelo teste de Tukey

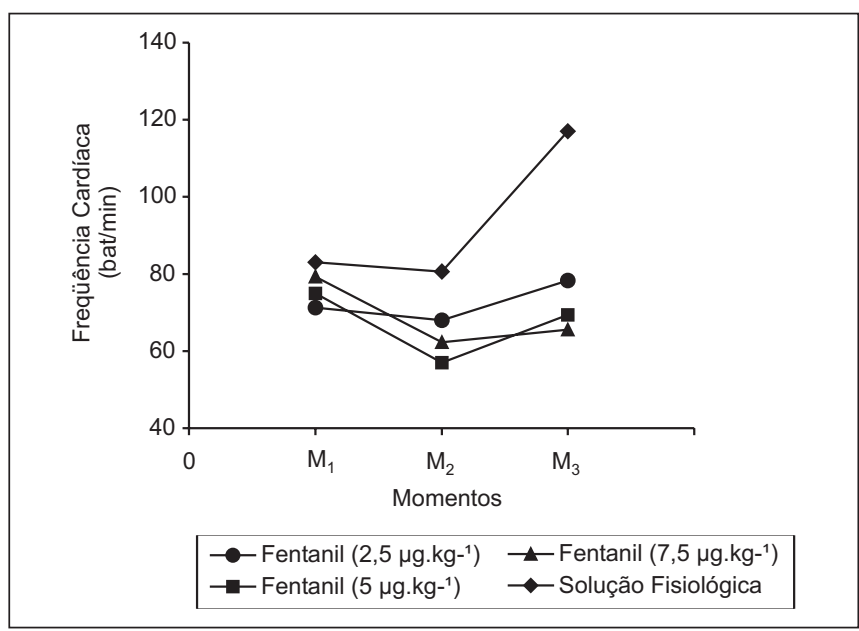

Figura 3 - Variações da Freqüência Cardíaca $\left(M_{1} \neq M_{3}\right.$ em $G 1, G 2, G 3$ e $\left.G 4-p<0,05\right)$ Análise estatística pelo teste de Tukey

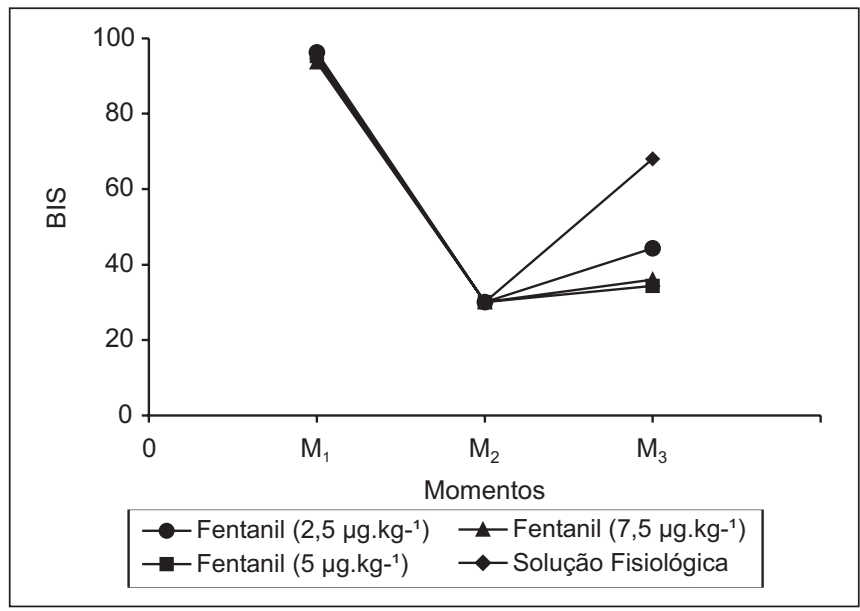

Figura 4 - Variação do Índice Bispectral

$\left(M_{2} \neq M_{3}\right.$ em G1 e G4. $M_{2}=M_{3}$ em G2 e G3 - $p>0,05$

Análise estatística pelo teste de Tukey

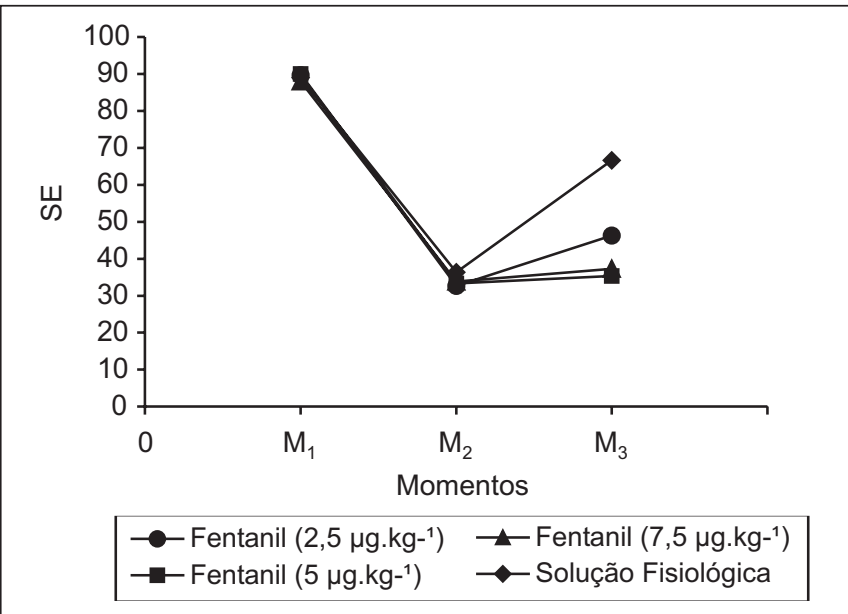

Figura 5 - Variação da SE

$\left(M_{2} \neq M_{3}\right.$ em G1 e G4 - $\left.p<0,05\right) \cdot M_{2}=M_{3}$ em G2 e G3 - $p>0,05$

Análise estatística pelo teste de Tukey

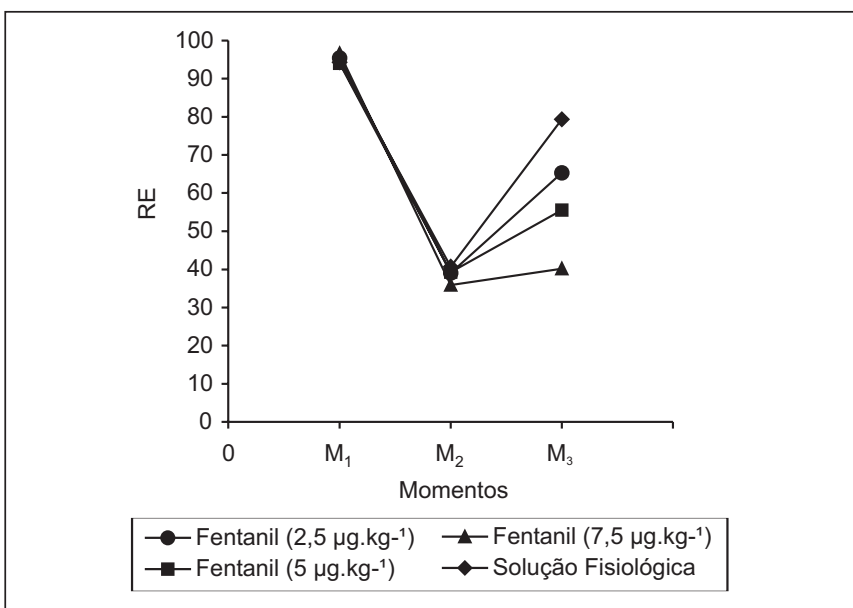

Figura 6 - Variações da RE

$(\mathrm{M} 2 \neq \mathrm{M} 2$ em G1, G2 e G4 - p < 0,05). M2 = M3 em G3- $p>0,05$ Análise estatística pelo teste de Tukey

\section{DISCUSSÃo}

Foi Claude Shannon ${ }^{7}$ quem, no final dos anos 40 , desenvolveu o conceito moderno de entropia "lógica" ou "de informação" como parte da medida de informação ou incerteza. A teoria da informação lidava com a recém-nascida ciência de comunicação de dados. A entropia de Shannon $(\mathrm{H})$ é dada pela seguinte equação (entropia canônica):

$H=-\Sigma p_{k} \log p_{k}$, onde $p_{k}$ são as probabilidades de um evento discreto $k$.

Trata-se de uma medida da dispersão dos dados. Dados com uma distribuição de probabilidade ampla e achatada apresentarão um elevado valor de entropia. Dados com uma distribuição estreita e em picos possuirão um baixo valor de enVol. 54, No 3, Maio - Junho, 2004 
tropia. Quando aplicada ao EEG, a entropia representa um descritor estatístico da variabilidade do sinal eletroencefalográfico (comparável à outros descritores, tal como a borda espectral ou a passagem a baixas freqüências que ocorre por ocasião da indução da anestesia geral).

Existem vários conceitos e técnicas analíticas direcionados à quantificação da irregularidade dos sinais estocásticos, tal como o EEG. A entropia é um desses conceitos. Enquanto conceito físico, a entropia é proporcional ao logaritmo do número de micro-estados disponíveis a um sistema termodinâmico, sendo, portanto, relacionada à quantidade de 'desordem' existente no sistema.

Contudo, o termo "desordem" é de difícil definição. Boltzman mostrou que a entropia termodinâmica podia ser definida precisamente como a constante de proporcionalidade $k$ (de Boltzman) multiplicada pelo logaritmo do número de micro-estados independentes $(\omega)$ disponíveis ao sistema (entropia microcanônica):

\section{$S=k \log (\omega)$}

Boltzman conseguiu explicar as mudanças nos macro-parâmetros observáveis (como, por exemplo, a temperatura) a partir das mudanças na energia cinética de uma coleção de moléculas individuais e, assim, tornou-se o pioneiro da ciência da mecânica estatística. A entropia termodinâmica tem uma base física bem estabelecida. É possível derivar a entropia de Shannon (ou entropia "de informação") $(H)$ da fórmula termodinâmica de Boltzman $(S)$. Deve-se deixar claro que a existência de uma analogia formal entre $\mathrm{He} S$ não implica que haja base material para uma equação entre $H$ e $S$ no que diz respeito à função cortical. Contudo, existem importantes indícios neurofisiológicos de que a utilidade dos estimadores de entropia de informação como medida da função cortical deve-se ao fato de que, à medida que o córtex passa à inconsciência, ocorre um verdadeiro decréscimo a nível neuronal no logaritmo do número de micro-estados acessíveis $(S)^{8,9}$. Assim, o termo "entropia" pode representar mais que uma mera medida estatística do padrão eletroencefalográfico; talvez, de alguma maneira, reflita verdadeiramente o fluxo de informação intra-cortical.

Entropia é o logaritmo do número de modos em que o micro-estado pode rearranjar-se e ainda produzir o mesmo macro-estado. A diferença entre a verdadeira entropia termodinâmica e as outras entropias de informação é que a distribuição da energia cinética das moléculas individuais não está necessariamente envolvida nos estimadores de entropia de informação. Abstraindo-se o termo 'energia' do contexto de calor e termodinâmica, ele pode denotar qualquer mudança na atividade das 'partículas' de que o sistema em observação é composto. A 'energia' representa, então, as mudanças no potencial da membrana piramidal cortical; essas mudanças produzem flutuações no potencial de campo local do córtex. Na teoria da informação, a primeira definição de entropia foi a de Shannon, em 1948. Depois, em 1984, Johnson e Shore ${ }^{10}$ a aplicaram ao espectro de potência de um sinal. Nesse con- texto, a entropia descreve a irregularidade, complexidade ou grau de incerteza de um sinal. Vejamos um exemplo simples: um sinal em que os valores seqüenciais são alternadamente de uma determinada magnitude fixa e depois de outra tem um valor de entropia de zero, ou seja, o sinal é completamente regular e totalmente previsível. Um sinal em que os valores seqüenciais são provenientes de um gerador de números aleatórios tem níveis maiores de complexidade e entropia. Um melhor entendimento clínico sobre eletromiografia de superfície, entropia de estado e entropia de resposta é de fundamental importância. A eletromiografia de superfície (EMGs) fornece a adição algébrica de atividade elétrica em uma população de fibras de músculo. Há uma relação direta entre amplitude EMGs e tensão de músculo durante a contração muscular isométrica (sem movimento) ${ }^{11}$. Em pacientes conscientes sem uso de agentes anestésicos, alta atividade tônica observada na eletromiografia, correlaciona-se positivamente com o nível de estímulo, vigilância ou estresse psicológico. Atividade fásica aumentada na EMGs está associada com períodos de estresse somático, por exemplo dor ${ }^{12}$. Na monitorização do paciente inconsciente, a EMGs deve ser mensurada na musculatura frontal, que tem um comprimento relativamente fixo, o qual diminui a influência potencialmente complicadora na variação do comprimento das fibras (contração isotônica). Esta musculatura é preferida, também, por causa de sua inervação pelas fibras eferentes viscerais especiais do nervo facial. É importante lembrar que esta musculatura é derivada dos arcos branquiais e estes são considerados formações viscerais.

AEMGs da musculatura frontal fornece uma simples e não invasiva medida de um aspecto do tônus autonômico. Contrações voluntárias e involuntárias da musculatura frontal são respostas representadas por inervações através de vias distintas, já que a atividade tônica(basal) e a atividade fásica (abruptamente aumentada) na EMGs podem ser diferentemente afetadas por níveis alterados de vigilância ou agentes bloqueadores neuromusculares ${ }^{13-15}$.

A diminuição da vigilância que acompanha a indução da anestesia geral é tipicamente associada com a dramática diminuição na atividade frontal tônica. Vários autores ${ }^{14,16,17}$ concluíram que aumento na atividade fásica na presença de drogas que deprimem a amplitude EMGs é um indicativo de anestesia inadequada. Além disso, todos estes investigadores observaram que a atividade fásica medida pela EMGs pode acorrer na presença de agentes bloqueadores neuromusculares.

Tem sido demonstrado que, pelo menos, três tipos fundamentalmente diferentes de estímulos - emoção, sons e isquemia - podem evocar um aumento fásico na amplitude EMG de superfície durante estados de baixa vigilância. AEMGs pode ter utilidade na titulação de opióides: músculos faciais não são somente voluntários, mas também inervados por centros emotivos localizados no tronco cerebral (relacionados a emoções/estresse). Embora a ativação eletromiográfica intra-operatória com o movimento do paciente seja um fenômeno quantal, pequenas mudanças na EMGs po- 
dem refletir inadequação analgésica (componente, sub-cortical com bloqueio inadequado). Mathews e col. ${ }^{18}$ mostraram que o uso de opióides após cirurgia diminuiu a EMGs. Além disso, a EMGs pode atuar mais rápido que o BIS em reações de despertar. Kern e col. ${ }^{19}$ concluíram que EMGs apresenta correspondência em resposta a estímulos nocivos aplicados a voluntários. Shander e col. ${ }^{20}$ também mostraram que EMGs podem predizer necessidade de analgésicos, permitindo controle mais efetivo intra-operatório da analgesia (componente sub-cortical). Lennon ${ }^{21}$ em trabalho intitulado Effect of partial neuromuscular blockade on intraoperative electromyography in patients undergoing resection of acoustic neuromas, concluiu que graus moderados de bloqueio neuromuscular podem ser alcançados sem comprometer a monitorização eletromiográfica do nervo facial. Edmonds ${ }^{15}$ mostrou que EMGs, em resposta ao estresse (dor), pode mensurar função do tronco cerebral a qual é independente do nível de consciência (córtex). Dutton ${ }^{22}$ em recente estudo, intitulado Craniofacial electromyogram activation response: another indicator of anesthetic depth, concluiu que a resposta eletromiográfica pode ser utilizada para estimar a profundidade anestésica.

Um biopotencial captado na região frontal de um paciente inclui um componente eletromiográfico significante criado pela atividade muscular. O sinal eletromiográfico tem um espectro amplo e semelhante a ruído e, durante a anestesia, predomina tipicamente nas freqüências acima de $30 \mathrm{~Hz}$. O componente do sinal eletroencefalográfico predomina nas freqüências inferiores (aproximadamente até $30 \mathrm{~Hz}$ ) contidas nos biopotenciais existentes nos eletrodos. Em freqüências mais elevadas, a potência eletroencefalográfica diminui exponencialmente.

O súbito aparecimento de dados de sinais eletroencefalográficos muitas vezes indica que o paciente está respondendo a alguns estímulos externos, como por exemplo, a estímulos dolorosos, ou seja, pode ser nocicepção em decorrência de algum evento cirúrgico. Respostas desse tipo podem ser o resultado de um nível insuficiente de anestésico. Se a estimulação continuar sem que sejam administrados quaisquer analgésicos adicionais, é muito provável que o nível de hipnose se torne mais superficial. Portanto, o sinal eletromiográfico pode dar uma indicação da iminência do despertar. Observe que, devido à freqüência mais elevada do sinal eletromiográfico, o tempo de amostragem pode ser significantemente mais curto do que para os dados de sinais eletroencefalográficos, que são de freqüência mais baixa. Com isso, os dados eletromiográficos podem ser calculados com freqüência maior de modo que o indicador diagnóstico geral possa indicar rapidamente as mudanças que ocorrerem no estado do paciente.

Para maior clareza deve-se considerar dois indicadores de entropia, um sobre a faixa de freqüência dominante do EEG somente e outro sobre a faixa completa de freqüências, incluindo componentes eletroencefalográficos e eletromiográficos. A 'entropia de situação' (SE) é calculada sobre a faixa de freqüência de $0,8 \mathrm{~Hz}$ a $32 \mathrm{~Hz}$. Ela inclui a parte eletroencefa- lográfica-dominante do espectro e, por conseguinte, reflete primariamente o estado cortical do paciente. As janelas de tempo para a SE são idealmente escolhidas para cada componente de freqüência específica, variando de $60 \mathrm{~s}$ a $15 \mathrm{~s}$. A 'entropia de resposta' (RE) é calculada sobre a faixa de freqüência de $0,8 \mathrm{~Hz}$ a $47 \mathrm{~Hz}$. Inclui a parte eletroencefalográfica-dominante e a parte eletromiográfico-dominante do espectro. As janelas de tempo para a RE são idealmente escolhidas para cada freqüência, a mais longa sendo igual a 15,36 s e a mais curta (aplicada a freqüências entre $32 \mathrm{~Hz}$ e $47 \mathrm{~Hz}$ ) sendo de 1,92 s.

É conveniente normalizar esses dois parâmetros de entropia de modo que a RE fique igual à SE sempre que a potência eletromiográfica (a soma da potência espectral entre $32 \mathrm{~Hz}$ e 47 $\mathrm{Hz}$ ) for igual a zero, pois assim a diferença entre RE e SE servirá como indicador da atividade eletromiográfica. Dar-se-á, à faixa de freqüência de $0,8 \mathrm{~Hz}$ a $32 \mathrm{~Hz}$ o nome de ' $\mathrm{R}_{\text {low' }}$ ', e à faixa de freqüência de $32 \mathrm{~Hz}$ a $47 \mathrm{~Hz}$ o de ' $R_{\text {high' }}$. Chama-se-á

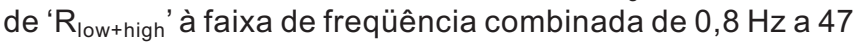
$\mathrm{Hz}$. Assim, quando os componentes espectrais dentro da $R_{\text {high }}$ são iguais a zero, os valores de entropia não-normalizados, $S\left[R_{\text {low }}\right]$ e $S N\left[R_{\text {low }}+R_{\text {high }}\right]$, irão coincidir, enquanto que para as entropias normalizadas obtém-se a desigualdade $S N\left[R_{\text {low }}\right]>S N\left[R_{\text {low }}+R_{\text {high }}\right]$. Aetapa de normalizaçãoé, portanto, redefinida para $S E$ da seguinte forma:

$$
S E=S_{N}\left[R_{\text {low }}\right]=\frac{S\left[R_{\text {low }}\right]}{\log \left(N\left[R_{\text {low }+ \text { high }}\right]\right)}=\frac{\log \left(N\left[R_{\text {low }}\right]\right)}{\log \left(N\left[R_{\text {low }+ \text { high }}\right]\right)} \cdot \frac{S\left[R_{\text {low }}\right]}{\log \left(N\left[R_{\text {low }}\right]\right)}
$$

Para a RE, o valor de entropia normalizado é calculado de acordo com a equação:

$$
R E=S_{N}\left[R_{\text {low }+ \text { high }}\right]=\frac{S\left[R_{\text {low }+ \text { high }}\right]}{\log \left(N\left[R_{\text {low }+ \text { high }}\right]\right)}
$$

Conseqüentemente, a $R E$ varia de 0 a 1 , enquanto que a $S E$ varia de 0 a $\log \left(N\left[R_{\text {low }}\right]\right) / \log \left(N\left[R_{\text {low+high }}\right]\right)<1$. Os dois valores de entropia coincidem quando $P\left(f_{i}\right)=0$ para todos os $f_{i}$ da fai$x a\left[R_{\text {high }}\right]$. Quando existe atividade eletromiográfica, os componentes espectrais na faixa $\left[R_{\text {high }}\right]$ diferem significantemente de zero, e a RE é superior à SE.

Com essas definições, a SE e a RE têm para o anestesiologista propósitos informativos distintos. A entropia de situação constitui uma quantidade suficientemente estável para que ele, consultando rapidamente um único número, possa ter uma idéia do estado cortical em que o paciente se encontra em qualquer dado momento. As janelas de tempo da SE são selecionadas de modo que as flutuações transitórias sejam retiradas dos dados. A entropia de resposta, por outro lado, reage rapidamente à mudanças. Os papéis distintos desses parâmetros são demonstrados, tipicamente, durante o despertar, quando, inicialmente, a RE aumenta juntamente com a ativação muscular, para ser seguida - poucos segundos depois - pela SE. 
A intubação traqueal representa um momento de grande estresse para o paciente, traduzido por alterações hemodinâmicas, endócrino-metabólicas ${ }^{23}$ (sub-corticais) e corticais (sistema nervoso central). Randel e col. ${ }^{24}$ relataram que pacientes ventilados com isoflurano em óxido nitroso e oxigênio, apresentaram ativação simpática representada por aumento na freqüência cardíaca sem alteração na resposta pressórica à intubação e que a medicação pré-anestésica com opióides, pode prevenir aumentos na concentração plasmática de adrenalina, porém não de noradrenalina, após laringoscopia e intubação. Outros autores avaliaram a influência de $2 \mu \mathrm{g} . \mathrm{kg}^{-1}$, por via venosa, de fentanil associado ao propofol na atenuação das respostas hemodinâmicas e corticais (através do BIS) à intubação, concluindo que esta técnica foi eficiente em bloquear as respostas hemodinâmicas, sem atenuar a resposta da atividade cortical cerebral ${ }^{25}$. Billare col. ${ }^{26}$ demonstraram que $2 \mu \mathrm{g} . \mathrm{kg}^{-1}$ de fentanil associado ao propofol diminuem a pressão sangüínea e a freqüência cardíaca em resposta à intubação. Neste estudo, comparouse a influência do sevoflurano isoladamente (G4) e sevoflurano associado ao fentanil (G1, G2 e G3) avaliando as respostas corticais cerebrais (BIS e SE), sub-corticais (RE) e hemodinâmicas (PAS, PAD e FC), assim como a resposta motora à intubação (clínica). Em relação à atividade cortical cerebral houve variações importantes no BIS entre os momentos $M_{2}$ a $M_{3}$, nos grupos: $G 1$ e $G 4$ com valores atingindo limites considerados entre consciência e inconsciência apenas no G4, podendo produzir o chamado estresse pós-traumático, sendo esta complicação mais provável quando o despertar é acompanhado de dor ${ }^{27}$. Os valores de SE evidenciaram trajetória semelhante à do BIS, na monitorização da atividade cortical cerebral. Todos os pacientes do G4 e $66 \%$ do G1 apresentaram resposta motora ao procedimento (IOT), o que não está de acordo com trabalho de Vernon e col. ${ }^{28}$, o qual evidencia que com valores de BIS abaixo de 40 , a probabilidade de movimento é praticamente nula tanto em técnicas anestésicas com o uso propofol/alfentanil como isoflurano/alfentanil. Vale ressaltar, entretanto, que o movimento é sub-cortical e BIS reflete diretamente o componente cortical e, apenas, indiretamente, o sub-cortical ${ }^{29-31}$. Os nossos resultados sugerem uma dissociação entre os níveis de hipnose e de analgesia em G1, G2 e G4, haja vista a presença de movimento à intubação nos grupos G1 e G4 e um gap maior que 10 no G2 (o que representou, também, entre os momentos $M_{2}$ e $M_{3}$, um $p<0,05 \%$, para os valores de RE). Apenas o G3 não mostrou diferença significativa entre os momentos $\mathrm{M}_{2}$ e $\mathrm{M}_{3}$ para a variável RE, o que pode refletir melhor controle anestésico. Nos pacientes do grupos: G1, G2 e G3, os parâmetros PAS, PAD e FC diminuíram significativamente de $M_{1}$ a $M_{3}$, onde $M_{3}$ sempre apresentou os mais baixos escores. Clinicamente, entretanto, estas variações não foram relevantes. No G4, não houve mudanças importantes nos parâmetros hemodinâmicos (PAS e PAD) entre $M_{1}$ e $M_{3}(p>0,05)$, excetuando-se apenas a FC $(p<0,05)$, com maiores valores registrados em $\mathrm{M}_{3}$. Não foram observadas respostas motoras às manobras de intubação orotraqueal nos grupos G2 e $\mathrm{G} 3$ o que poderia sugerir a dose de $5 \mu \mathrm{g} \cdot \mathrm{kg}^{-1}$ como ideal para
IOT. Contudo, a entropia de resposta mostrou que o mais eficiente bloqueio da atividade sub-cortical aconteceu no G3. Assim, este estudo mostrou que o sevoflurano isoladamente, associado a $2,5 \mu \mathrm{g} \cdot \mathrm{kg}^{-1}$ ou $5 \mu \mathrm{g} \cdot \mathrm{kg}^{-1}$ de fentanil não bloqueou efetivamente as respostas dos componentes cortical e sub-cortical do SNC, fato observado com a dose de 7,5 $\mu \mathrm{g} . \mathrm{kg}^{-1}$ com a qual ocorreu melhor controle destes componentes anestésicos.

\section{Entropy: A New Method of Measuring Depth of Anesthesia. Comparative Study with Bispectral Index during Clinical Evaluation in Tracheal Intubation of Patients Anesthetized with Sevoflurane}

\author{
Rogean Rodrigues Nunes, TSA, M.D.
}

\section{INTRODUCTION}

Spectral entropy, a new EEG analysis method based on the quantification of EEG chaos, was developed to monitor anesthetic depth ${ }^{1,2}$. As with BIS, spectral entropy also uses a 0 to 100 scale. In addition, it involves two distinct types of analysis: state entropy, which includes low frequency signals up to $32 \mathrm{~Hz}$ (quantifies cortical cerebral activity - hypnosis), and response entropy, which includes signals up to $47 \mathrm{~Hz}$ (evaluates surface EMG activity - sub-cortical component).

This study aimed at comparing entropy-derived values (both state and response entropy) to BIS-derived values to evaluate cortical and sub-cortical (autonomic and somatic) responses during tracheal intubation (TI) in patients submitted to general anesthesia with sevoflurane associated or not to fentanyl.

\section{METHODS}

After the Institution's Ethics Committee approval, participated in this randomized double-blind study 36 patients of both genders, aged 20 to 44 years, physical status ASA I, Mallampati class I, normal head extension and body mass index between 21 and 28. Exclusion criteria were patients under drugs knowingly affecting EEG and sevoflurane MAC, drug abusers, use of sedatives in the last 48 hours or neuromuscular diseases. Patients were induced with sevoflurane and distributed in four groups of nine patients (G1, G2, G3 and G4, according to opioid (fentanyl) dose. Anesthesia was induced with sevoflurane by tidal volume technique, with initial vaporizer percentage demand established in $5 \%$ of $\mathrm{O}_{2}$ flow equal to 4 L. $\mathrm{min}^{-1}$ until reaching bispectral index $=65^{3}$ (threshold between consciousness and uncon- 
sciousness), moment in which fentanyl was injected in the following doses: G1 $\left(2.5 \mu \mathrm{g} . \mathrm{kg}^{-1}\right), \mathrm{G} 2\left(5 \mu \mathrm{g} . \mathrm{kg}^{-1}\right), \mathrm{G} 3(7.5$ $\mu \mathrm{g} . \mathrm{kg}^{-1}$ ) and G4 (saline solution). Tracheal intubation was performed 5 minutes after by the same anesthesiologist and with just one type of blade (Macintosh) ${ }^{4}$, with anesthetic (sevoflurane) expired concentration adjusted at this time to maintain $\mathrm{BIS}=30$ (stabilized in 5 minutes), with manual ventilation under mask.

Three successive moments were evaluated for each group $\left(M_{1}=\right.$ immediately before anesthetic induction; $M_{2}=$ immediately before tracheal intubation; and $\mathrm{M}_{3}=$ one minute after tracheal intubation). The following parameters were evaluated: bispectral index (BIS), state entropy (SE), response entropy (RE), sevoflurane expired concentration (EC), systolic blood pressure (SBP), diastolic blood pressure (DBP) and heart rate (HR). Skeletal muscle motor response was also recorded as adequate anesthetic depth parameter ${ }^{5}$ during tracheal intubation. Clinically accepted lowest hemodynamic parameters limits were: SBP: $75 \mathrm{mmHg}$ and DBP: $55 \mathrm{mmHg}$. Upper limits were considered clinically significant when above $30 \%$ of $M_{1}$ values. As to $H R$, values $30 \%$ above $M_{1}$ were also considered clinically significant. As to entropy values, state entropy varies 0 to 91 (between 40 and 60 during adequate anesthesia) and response entropy varies 0 to 100 (with adequate values between 40 and 60 ), being its values equal to or higher than SE when there is EMG activation. This activation is seen as a gap between $R E$ and $S E{ }^{6}$ which should be maintained below 10. Electrodes (BIS and entropy) were placed on fronto-temporal regions so that BIS would evaluate left channel and entropy would evaluate right channel, being initial measurement recorded after asking patients to remain with eyes closed. No patient was premedicated.

Results were submitted to statistical analysis through Analysis of Variance. Tukey's test was applied among levels of involved factors, considering significant $p<0.05$.

\section{RESULTS}

Demographics data are shown in table I.

Table I - Demographics Data (Mean \pm SD)

\begin{tabular}{lcccc}
\hline Variables & \multicolumn{4}{c}{ Groups } \\
\hline & $\begin{array}{c}\text { Fentanyl } \\
(2 \mu \mathrm{g})\end{array}$ & $\begin{array}{c}\text { Fentanyl } \\
(5 \mu \mathrm{g})\end{array}$ & $\begin{array}{c}\text { Fentanyl } \\
(7.5 \mu \mathrm{g})\end{array}$ & $\begin{array}{c}\text { Saline } \\
\text { Solution }\end{array}$ \\
\hline Weight $(\mathrm{kg})$ & $56.64 \pm 2.61$ & $58.80 \pm 3.17$ & $61.00 \pm 4.67$ & $49.55 \pm 5.14$ \\
Age (years) & $27.30 \pm 7.10$ & $22.34 \pm 5.44$ & $27.99 \pm 6.90$ & $24.32 \pm 4.12$ \\
Height $(\mathrm{m})$ & $1.61 \pm 0.05$ & $1.56 \pm 0.09$ & $1.53 \pm 0.06$ & $1.58 \pm 0.12$ \\
\hline
\end{tabular}

Mean SBP, DBP and HR in the three studied moments are shown in table II and figures 1, 2 and 3 . It is observed statistically significant decrease $(p<0.05)$ in moments $M_{1}$ and $M_{3}$ only, for G1, G2 and G3. There have been no statistically significant SBP changes in $\mathrm{G} 4$. In comparing $\mathrm{M}_{1}$ and $\mathrm{M}_{3}$, there has been statistically significant difference in DBP in $\mathrm{G} 1$ and G4 only ( $p<0.05$ ), which has not been observed for $G 2$ and G3. In comparing $M_{1}$ and $M_{3}$, there have been statistically significant HR differences for all groups. Clinically, however, according to prefixed criteria, changes were not significant, except for HR increase in $\mathrm{G} 4$. There has been motor reaction to $\mathrm{TI}$ in $66 \%$ of $\mathrm{G} 1$ patients. In G4, all patients had motor reaction to intubation maneuvers.

Mean BIS, SE and RE values are shown in table III and figures 4, 5 and 6. It is observed that BIS has not shown statistically significant differences in $\mathrm{G} 2$ and $\mathrm{G} 3$ between moments $M_{1}$ and $\mathrm{M}_{3}$, being observed statistically significant BIS increase between M2 and M3 for G1 and G4 ( $p<0.05)$, reaching threshold values between consciousness and unconsciousness (above 68) (Figure 4).

SE has not shown statistically significant difference between $M_{2}$ and $M_{3}$ when $G 2$ and $G 3$ were compared, being observed statistically significant differences between $M_{2}$ and $M_{3}$ for $G 1$ and G4 (changes parallel to BIS).

Table II - Systolic Blood Pressure, Diastolic Blood Pressure, Heart Rate in the Four Groups and Studied Moments (Mean \pm SD)

\begin{tabular}{|c|c|c|c|c|c|}
\hline \multirow[t]{2}{*}{ Moments } & \multirow[t]{2}{*}{ Variables } & \multicolumn{4}{|c|}{ Groups } \\
\hline & & $\begin{array}{c}\text { G1 = Fentanyl } \\
\left(2.5 \mu \mathrm{gg}^{-1}\right)\end{array}$ & $\begin{array}{c}\mathrm{G} 2=\text { Fentanyl } \\
\left(5 \mu \mathrm{g} \cdot \mathrm{kg}^{-1}\right)\end{array}$ & $\begin{array}{c}\text { G3 = Fentanyl } \\
\left(7.5 \mu \mathrm{ggg}^{-1}\right)\end{array}$ & G4 = Saline Solution \\
\hline \multirow[t]{3}{*}{$M_{1}$} & SBP & $121.30 \pm 13.90$ & $112.67 \pm 10.07$ & $125.00 \pm 6.56$ & $131.67 \pm 9.04$ \\
\hline & DBP & $81.00 \pm 8.44$ & $71.00 \pm 2.00$ & $68.33 \pm 4.60$ & $64.31 \pm 6.63$ \\
\hline & $\mathrm{HR}$ & $71.30 \pm 10.69$ & $75.00 \pm 15.70$ & $79.32 \pm 6.50$ & $83.00 \pm 8.70$ \\
\hline \multirow[t]{3}{*}{$\mathrm{M}_{2}$} & SBP & $83.00 \pm 10.23$ & $79.35 \pm 4.15$ & $82.20 \pm 5.19$ & $84.65 \pm 4.44$ \\
\hline & DBP & $56.00 \pm 4.75$ & $57.67 \pm 3.32$ & $54.00 \pm 6.00$ & $53.66 \pm 6.12$ \\
\hline & $\mathrm{HR}$ & $68.00 \pm 9.17$ & $57.00 \pm 3.00$ & $62.30 \pm 1.53$ & $80.60 \pm 14.15$ \\
\hline \multirow[t]{3}{*}{$\mathrm{M}_{3}$} & SBP & $100.33 \pm 6.01$ & $96.60 \pm 4.31$ & $90.00 \pm 10.58$ & $129.00 \pm 12.06$ \\
\hline & DBP & $79.00 \pm 9.52$ & $63.33 \pm 10.53$ & $60.00 \pm 10.49$ & $82.67 \pm 12.62$ \\
\hline & $H R$ & $78.33 \pm 10.20$ & $69.44 \pm 9.18$ & $65.60 \pm 4.60$ & $117.00 \pm 10.14$ \\
\hline
\end{tabular}

$\mathrm{SBP}=\mathrm{mmHg}, \mathrm{DBP}=\mathrm{mmHg}, \mathrm{HR}=\mathrm{bpm}$ 
ENTROPY: A NEW METHOD OF MEASURING DEPTH OF ANESTHESIA. COMPARATIVE STUDY WITH BISPECTRAL INDEX DURING CLINICAL EVALUATION IN TRACHEAL INTUBATION OF PATIENTS ANESTHETIZED WITH SEVOFLURANE

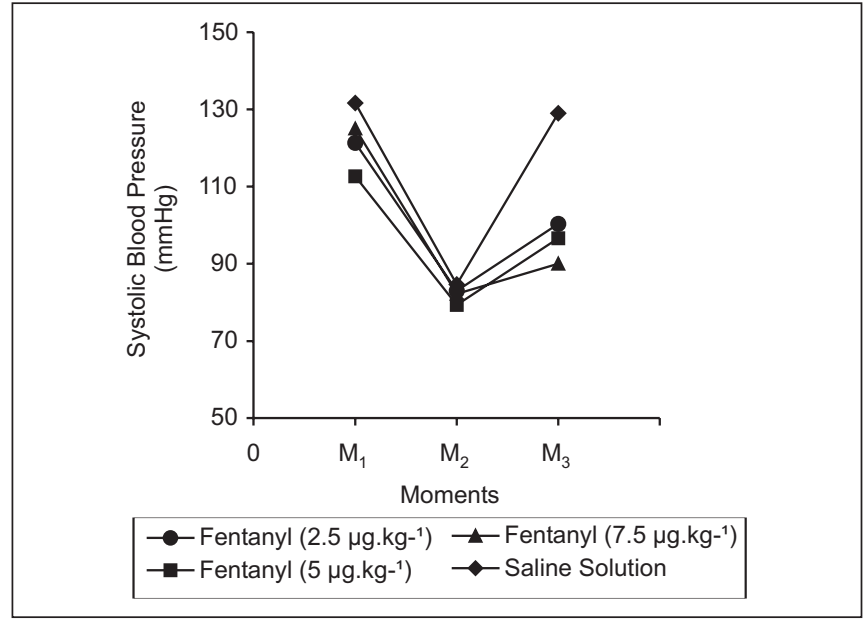

Figure 1 - Systolic Blood Pressure Variation $(\mathrm{mmHg})$ $\left(M_{1} \neq M_{3}\right.$ in $G 1, G 2$ and $\left.G 3-p<0.05 . M_{1}=M_{3}\right)$ in $\left.G 4-p>0.05\right)$ Tukey's test

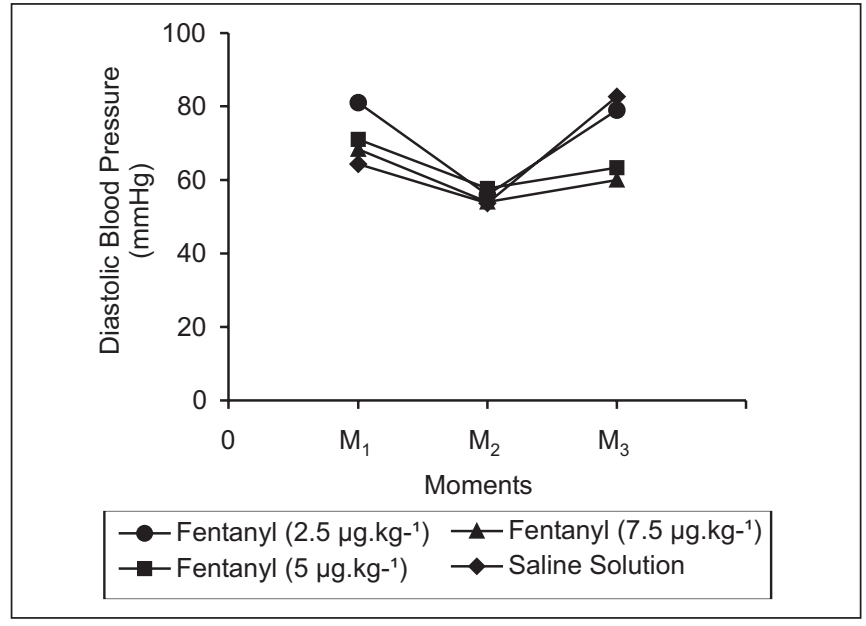

Figure 2 - Diastolic Blood Pressure Variation $(\mathrm{mmHg})$ $\left(M_{1} \neq M_{3}\right.$ in $G 1$ and $G 4-p<0.05 . M_{1}=M_{3}$ in $G 2$ e G3 $\left.-p>0.05\right)$ Tukey's test

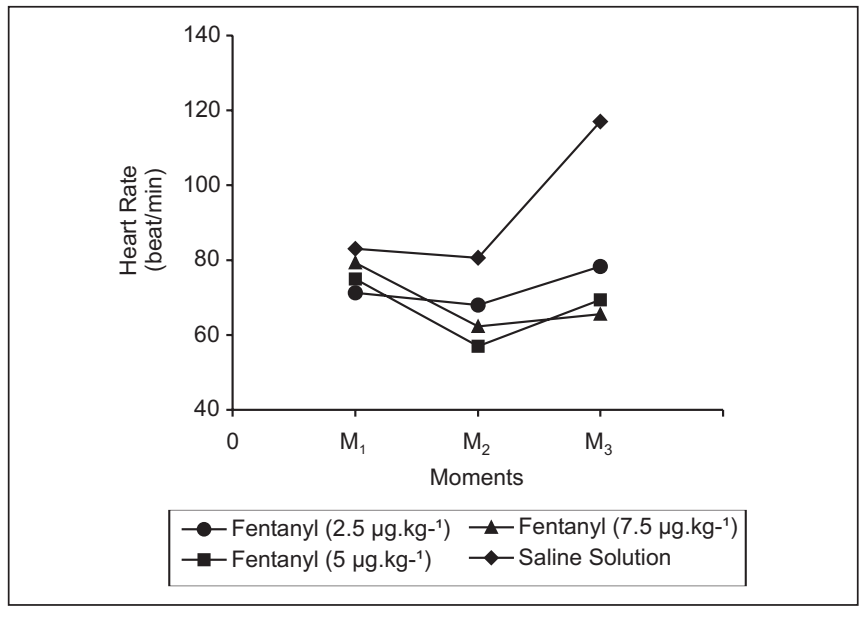

Figure 3 - Heart Rate Variations

$\left(M_{1} \neq M_{3}\right.$ in $\mathrm{G} 1, \mathrm{G} 2, \mathrm{G} 3$ and $\left.\mathrm{G} 4-\mathrm{p}<0.05\right)$ Tukey's test

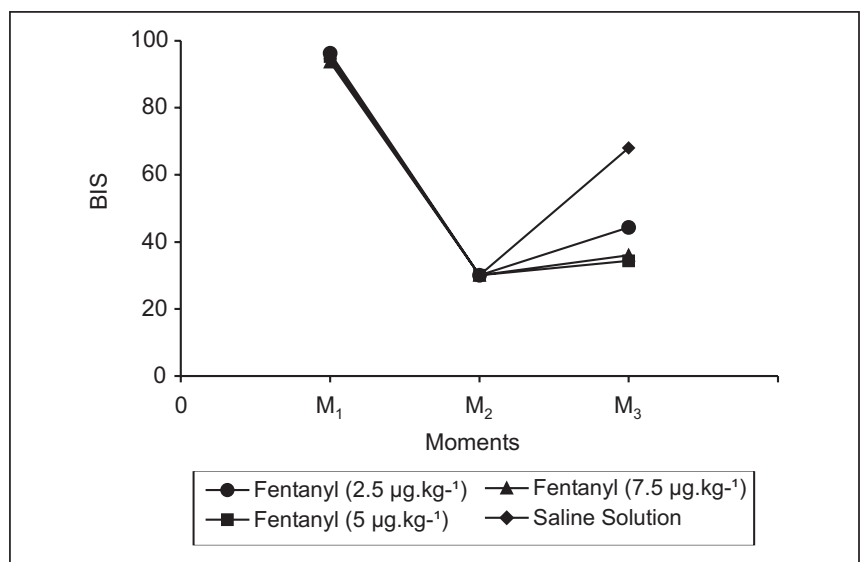

Figure 4 - Bispectral Index Variation

$\left(M_{2} \neq M_{3}\right.$ in $G 1$ and $G 4 . M_{2}=M_{3}$ in $G 2$ and $G 3-p>0.05$ Tukey's test

Table III - BIS, SE, RE and CE in the Four Groups and Studied Moments (Mean \pm SD)

\begin{tabular}{|c|c|c|c|c|c|}
\hline \multirow[t]{2}{*}{ Moments } & \multirow[t]{2}{*}{ Variables } & \multicolumn{4}{|c|}{ Groups } \\
\hline & & $\begin{array}{c}\text { G1 = Fentanyl } \\
\left(2.5 \mu \mathrm{g} \cdot \mathrm{kg}^{-1}\right)\end{array}$ & $\begin{array}{c}\mathrm{G} 2=\text { Fentanyl } \\
\left(5 \mu \mathrm{g} \cdot \mathrm{kg}^{-1}\right)\end{array}$ & $\begin{array}{c}\text { G3 = Fentanyl } \\
\left(7.5 \mu \mathrm{gg}^{-1}\right)\end{array}$ & G4 = Saline Solution \\
\hline \multirow[t]{4}{*}{$\mathrm{M}_{1}$} & BIS & $96.33 \pm 2.00$ & $95.30 \pm 1.43$ & $93.68 \pm 1.58$ & $94.67 \pm 3.00$ \\
\hline & SE & $89.67 \pm 2.28$ & $90.00 \pm 1.25$ & $88.00 \pm 2.58$ & $89.00 \pm 1.61$ \\
\hline & RE & $95.40 \pm 3.10$ & $94.00 \pm 3.42$ & $96.67 \pm 0.84$ & $94.32 \pm 3.6$ \\
\hline & CE & $0.00 \pm 0.00$ & $0.00 \pm 0.00$ & $0.00 \pm 0.00$ & $0.00 \pm 0.00$ \\
\hline \multirow[t]{4}{*}{$M_{2}$} & BIS & $30.00 \pm 0.00$ & $30.00 \pm 0.00$ & $30.00 \pm 0.00$ & $30.00 \pm 0.00$ \\
\hline & SE & $32.67 \pm 3.00$ & $33.33 \pm 3.81$ & $33.87 \pm 2.69$ & $36.33 \pm 2.60$ \\
\hline & RE & $39.07 \pm 1.46$ & $39.27 \pm 2.20$ & $35.90 \pm 1.70$ & $40.67 \pm 1.20$ \\
\hline & CE & $3.83 \pm 0.53$ & $3.57 \pm 0.40$ & $4.40 \pm 0.54$ & $4.62 \pm 0.61$ \\
\hline \multirow[t]{3}{*}{$\mathrm{M}_{3}$} & BIS & $44.33 \pm 2.18$ & $34.33 \pm 3.60$ & $36.00 \pm 4.50$ & $68.00 \pm 5.6$ \\
\hline & SE & $46.33 \pm 2.75$ & $35.32 \pm 4.51$ & $37.30 \pm 2.81$ & $66.66 \pm 3.02$ \\
\hline & RE & $65.27 \pm 2.16$ & $55.50 \pm 2.41$ & $40.21 \pm 2.80$ & $79.34 \pm 4.57$ \\
\hline
\end{tabular}




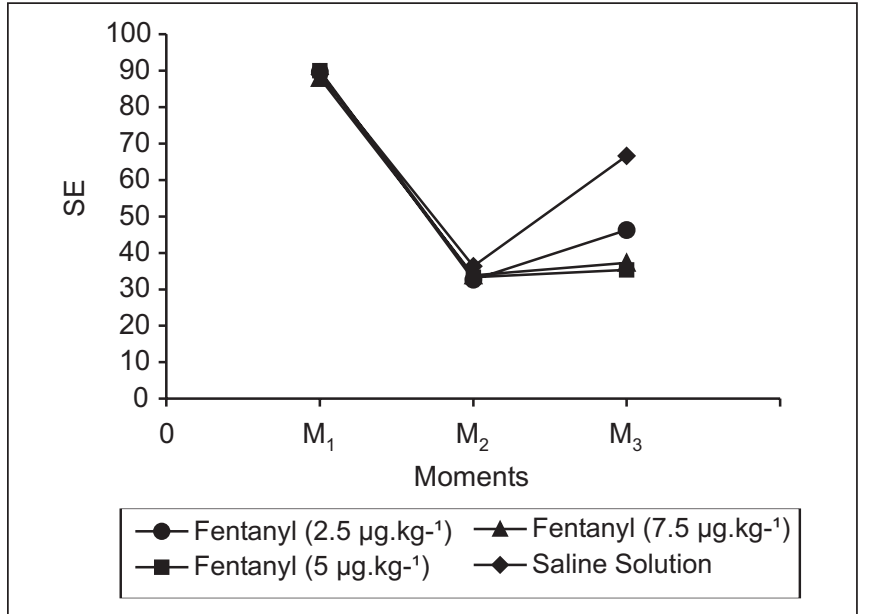

Figure 5 - SE Variation $\mathrm{SE}\left(\mathrm{M}_{2} \neq \mathrm{M}_{3}\right.$ in $\mathrm{G} 1$ and $\left.\mathrm{G} 4-\mathrm{p}<0.05\right)$ $M_{2}=M_{3}$ in $G 2$ and $G 3-p>0.05$ Tukey's test

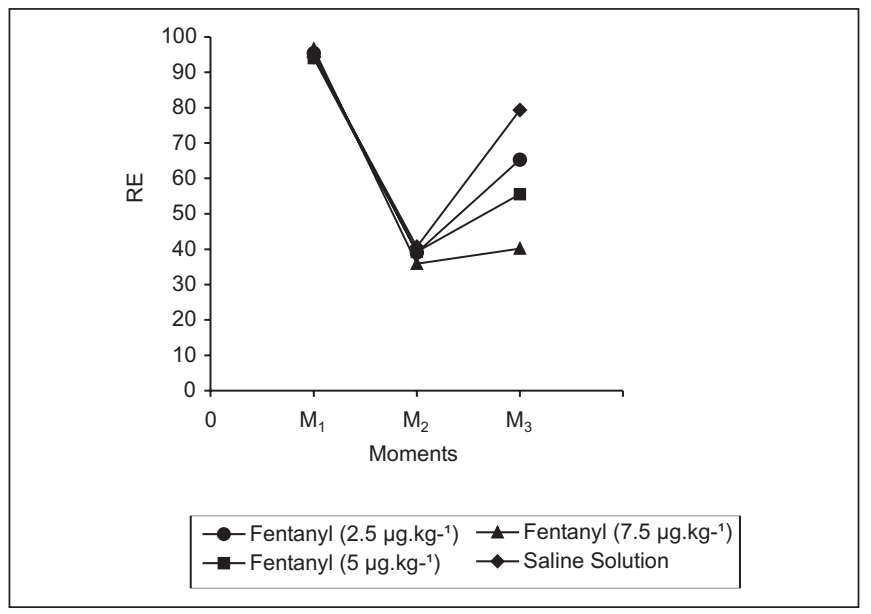

Figure 6 - RE Variations $\left(M_{2} \neq M_{2}\right.$ in $G 1, G 2$ and $\left.G 4-p<0.05\right)$ $\mathrm{M} 2=\mathrm{M} 3$ in $\mathrm{G} 3-\mathrm{p}>0.05$

Tukey's test

There have been, however, statistically significant RE increases between $M_{1}$ and $M_{3}$ for $G 1, G 2$ and $G 4$. For $G 3, p$ value was $>0.05$ and SE-RE gap was lower than 10 between $\mathrm{M}_{2}$ and $\mathrm{M}_{3}$.

\section{DISCUSSION}

It has been Claude Shannon ${ }^{7}$ who, in the late 1940 s, has developed the modern concept of "logic" or "information" entropy as part of information or uncertainty measurement. Information theory dealt with the newly born data communication science. Shannon's entropy $(H)$ is given by the following equation (canonic entropy):

$H=-\Sigma p_{k} \log p_{k}$, where $p_{k}$ are the probabilities of a discrete event $k$.
It is a data dispersion measurement. Data with wide and flat probability distribution will have a high entropy value. Data with narrow and peaked distribution will have a low entropy value. When applied to EEG, entropy is a statistical descriptor of EEG signal variability (comparable to other descriptors, such as spectral edge or low frequency passages during general anesthetic induction).

There are several concepts and analytical techniques aimed at quantifying stochastic signals irregularities, such as EEG. Entropy is one of those concepts. While physical concept, entropy is proportional to the logarithm of the number of available microstates to a thermodynamic system, thus being related to the amount of "disorder" existing in the system. However, the word "disorder" is difficult to define. Boltzman has shown that thermodynamic entropy could be accurately defined as the proportionality constant $k$ (Blotzman's) multiplied by the logarithm of the number of independent microstates $(\omega)$ available to the system (microcanonic entropy):

$$
S=k \log (\omega)
$$

Boltzman was able to explain changes in observable macro-parameters (such as temperature) as from kinetic energy changes of a collection of individual molecules and has become the pioneer of statistical mechanics science. Thermodynamic entropy has a well-established physical basis. It is possible to derive Shannon's entropy (or "information" entropy) $(H)$ from Boltzman's thermodynamic formula $(S)$. But it must be clearly stated that the existence of a formal analogy between $\mathrm{H}$ and $\mathrm{S}$ does not imply the existence of material basis for an equation between $H$ and $S$ with regard to cortical function. There are, however, major neurophysiological clues that the usefulness of information entropy estimators as measure of cortical function is due to the fact that, as cortex goes from consciousness to unconsciousness, there is a true neuronal decrease in the logarithm of the number of accessible microstates $(S)^{8,9}$. So, the word "entropy" may represent more than a simple statistical measurement of EEG pattern; may be, in a way, it would truly reflect intra-cortical information flow.

Entropy is the logarithm of the number of modes in which microstates may rearrange and still produce the same macrostate. The difference between true thermodynamic entropy and other information entropies is that kinetic energy distribution of individual molecules is not necessarily involved with information entropy estimators. By abstracting the word "energy" for the concept of heat and thermodynamics, it could reflect any change in the activity of "particles" making up the observed system. "Energy", then, represents changes in cortical pyramidal membrane potential; these changes promote potential local cortex field fluctuations. Shannon was the first to define entropy in the information theory, in 1948. Then, in 1984, Johnson and Shore ${ }^{10}$ have applied it to the power spectrum of a signal. Within this context, entropy describes irregularity, complexity or level of uncertainty of a signal. Let's see a simple example: a signal in which Vol. 54, N 3, Maio - Junho, 2004 
sequential values are alternately of one certain fixed magnitude and then of other has zero entropy value, that is, signal is totally regular and predictable. A signal with sequential values generated by a random numbers generator has higher complexity and entropy levels. Better clinical understanding of surface electromyography, state entropy and response entropy is critical, that is: surface electromyography $\left(E M G_{S}\right)$ gives the algebraic sum of electric activity in a population of muscle fibers. There is a direct ratio between $\mathrm{EMG}_{\mathrm{S}}$ amplitude and muscle stress during isometric muscle contraction (without movement) ${ }^{11}$. In conscious patients without anesthetics, high tone activity observed by electromyography is positively correlated to the level of stimulation, alertness or psychological stress. Increased EMGs phase activity is associated to periods of somatic stress, for example pain ${ }^{12}$. For unconscious patients monitoring, EMGs should be measured on frontal muscles, which have a relatively fixed length, thus minimizing the potentially complicating influence of fiber length variations (isotonic contraction). These muscles are also preferred for being innervated by special visceral efferent fibers of the facial nerve. It is important to remind that these muscles are derived from branchial arches which are considered visceral formations.

Frontal muscles $E M G_{S}$ provides a simple and noninvasive measurement of an autonomic tone aspect. Voluntary and involuntary frontal muscles contractions are responses represented by innervations through different pathways, since $E M G_{S}$ tone activity (baseline) and phase activity (abruptly increased) may be differently affected by abnormal alertness levels or by neuromuscular blockers ${ }^{13-15}$.

Decreased alertness following general anesthetic induction is typically associated to dramatic frontal tone activity decrease. Several authors ${ }^{14,16,17}$ have concluded that increased phase activity in the presence of drugs depressing $E M G_{S}$ amplitude is an indicator of inadequate anesthesia. In addition, these authors have also observed that $\mathrm{EMG}_{\mathrm{S}}$-measured phase activity may be observed in the presence of neuromuscular blockers.

It has been shown that at least three fundamentally different types of stimulations - emotion, sounds and ischemia - may evoke surface EMG amplitude phase increase during stages of decreased alertness.

$\mathrm{EMG}_{\mathrm{S}}$ may be useful for opioids titration: facial muscles are not only voluntary, but also innervated by emotive centers located in brainstem (related to emotions/stress). Although intraoperative EMG activation by patients' movement is a quantal phenomenon, minor $\mathrm{EMG}_{\mathrm{S}}$ changes may reflect analgesic inadequacy (sub-cortical component with inadequate block). Mathews et al. ${ }^{18}$ have shown that postoperative opioids decrease $E M G_{S}$. In addition, $E M G_{S}$ may act faster than BIS in emergence reactions. Kern et al. ${ }^{19}$ have concluded that EMGs has correspondence in response to noxious stimulations applied to volunteers. Shander et al. ${ }^{20}$ have also shown that $E M G_{S}$ may predict analgesic requirements, providing more effective intraoperative analgesic control (sub-cortical component). Lennon ${ }^{21}$, in a study called Effect of partial neuromuscular blockade on intraoperative electromyography in patients undergoing resection of acoustic neuromas, has concluded that moderate neuromuscular block levels may be reached without impairing facial nerve EMG monitoring. Edmonds ${ }^{15}$ has shown that $E M G_{S}$ in response to stress (pain) may measure brainstem function which is independent of consciousness level (cortex). Dutton 22 in a recent study called Craniofacial electromyogram activation response: another indicator of anesthetic depth, has concluded that EMG response may be used to estimate anesthetic depth.

A biopotential captured on patient's frontal region includes significant EMG component created by muscle activity. EMG signal has a wide spectrum similar to noise and, during anesthesia, it typically predominates in frequencies above $30 \mathrm{~Hz}$. EEG signal component predominates in lower frequencies (up to approximately $30 \mathrm{~Hz}$ ) contained in electrodes biopotentials. At higher frequencies, EEG power is exponentially decreased.

The sudden appearance of EEG signal data very often indicates that patient is responding to some external stimulation, such as painful stimulations, that is, it may be nociception as a consequence of a surgical event. These responses may be result of inadequate anesthetic level. If stimulation continues without the administration of additional analgesics, it is very possible that hypnosis will become more superficial. So, EMG signal may be an indication of emergence. Note that, due to higher EMG signal frequency, sampling time may be significantly shorter than for EEG signal data which are of lower frequency. With this, EMG data may be calculated with higher frequency so that the general diagnostic indicator may rapidly indicate changes in patient's status.

For better clarity, two entropy indicators should be considered, one on EEG-dominant frequency range and the other on complete frequencies range, including EEG and EMG components. "State" entropy (SE) is calculated on the frequency range $0.8 \mathrm{~Hz}$ to $32 \mathrm{~Hz}$. It includes the EEG-dominant part of the spectrum and primarily reflects patients' cortical status. Time windows for SE are ideally chosen for each specific frequency component and vary $60 \mathrm{~s}$ to $15 \mathrm{~s}$. "Response entropy" is calculated on frequency range $0.8 \mathrm{~Hz}$ to $47 \mathrm{~Hz}$, including EEG-dominant and EMG-dominant part of the spectrum. Time windows for RE are ideally chosen for each frequency, being the longest $=15.36 \mathrm{~s}$ and the shortest $($ applied to frequencies between $32 \mathrm{~Hz}$ and $47 \mathrm{~Hz}$ ) $=1.92 \mathrm{~s}$.

It is desirable to normalize both entropy parameters so that RE equals SE whenever EMG power (sum of spectral power between $32 \mathrm{~Hz}$ and $47 \mathrm{~Hz}$ ) equals zero, because this way, the difference between RE and SE will be an indicator of EMG activity. Here, frequency range $0.8 \mathrm{~Hz}$ to $32 \mathrm{~Hz}$ will be called $\mathrm{R}_{\text {low }}$ and frequency range $32 \mathrm{~Hz}$ to $47 \mathrm{~Hz}$ will be called $\mathrm{R}_{\text {high. }}$. Combined frequency range $0.8 \mathrm{~Hz}$ to $47 \mathrm{~Hz}$ will be called $\mathrm{R}_{\text {low }}$ high $/$. So, when spectral components within $R_{\text {high }}$ equal zero, non-normalized entropy values $S\left[R_{\text {low }}\right]$ and $S N\left[R_{\text {low }}+R_{\text {high }}\right]$ 
will coincide, while for normalized entropies there is the inequality $\mathrm{SN}\left[\mathrm{R}_{\text {low }}\right]>\mathrm{SN}\left[\mathrm{R}_{\text {low }}+\mathrm{R}_{\text {high }}\right]$. Normalization stage, then, is redefined for SE as follows:

$$
S E=S_{N}\left[R_{\text {low }}\right]=\frac{S\left[R_{\text {low }}\right]}{\log \left(N\left[R_{\text {low }+ \text { high }}\right]\right)}=\frac{\log \left(N\left[R_{\text {low }}\right]\right)}{\log \left(N\left[R_{\text {low }+ \text { high }}\right]\right)} \cdot \frac{S\left[R_{\text {low }}\right]}{\log \left(N\left[R_{\text {low }}\right]\right)}
$$

For RE, normalized entropy value is calculated according to equation:

$$
R E=S_{N}\left[R_{\text {low }+ \text { high }}\right]=\frac{S\left[R_{\text {low }+ \text { high }}\right]}{\log \left(N\left[R_{\text {low }+ \text { high }}\right]\right)}
$$

As a consequence, $R E$ varies 0 to 1 , while $S E$ varies 0 to $\log \left(N\left[R_{\text {low }}\right]\right) / \log \left(N\left[R_{\text {low+high }}\right]\right)<1$. Both entropy values coincide when $P\left(f_{i}\right)=0$ for all $f_{i}$ of the $\left[R_{\text {high }}\right]$ range. When there is EMG activity, spectral components in the range $\left[R_{\text {high }}\right]$ are significantly different from zero and RE is higher than SE. With these definitions, SE and RE have different information purposes for anesthesiologists. State entropy is a sufficiently stable quantity for the anesthesiologist, in rapidly checking a single number, to have an idea of patient's cortical status in any given moment. Time windows for SE are selected so that transient fluctuations are removed from data. Response entropy, on the other hand, rapidly reacts to changes. Different roles of such parameters are typically shown during emergence when first there is RE increase together with muscle activation, to be followed - some seconds after - by SE increase.

Tracheal intubation is a moment of major stress for patients, translated by hemodynamic, endocrine-metabolic ${ }^{23}$ (sub-cortical) and cortical (central nervous system) changes. Randel et al. ${ }^{24}$ have reported that patients ventilated with isoflurane in nitrous oxide and oxygen have presented sympathetic activation represented by heart rate increase without changes in pressure response to intubation, and premedication with opioids may prevent plasma epinephrine, but not norepinephrine, concentration increase after laryngoscopy and intubation. Other authors have evaluated the influence of intravenous $2 \mu \mathrm{g} . \mathrm{kg}^{-1}$ fentanyl associated to propofol in attenuating hemodynamic and cortical responses (through BIS) to intubation and have concluded that this technique has been effective in blocking hemodynamic responses without attenuating brain cortical activity response ${ }^{25}$. Billar et al. ${ }^{26}$ have shown that $2 \mu \mathrm{g} . \mathrm{kg}^{-1}$ fentanyl associated to propofol have decreased blood pressure and heart rate in response to intubation. In our study, we have compared the influence of sevoflurane alone (G4) and of sevoflurane associated to fentanyl (G1, G2 and G3), evaluat- ing brain cortical (BIS and SE) and sub-cortical (RE), and hemodynamic (SBP, DBP and HR) responses, as well as motor response to intubation (clinical). As to brain cortical activity, there have been major BIS variations between moments $M_{2}$ and $M_{3}$ in $G 1$ and $G 4$, with values reaching thresholds between consciousness and unconsciousness only in G4, and being able to produce the so-called post-trauma stress, being this complication most likely when emergence is followed by pain ${ }^{27}$. SE values were similar to BIS in monitoring brain cortical activity. All G4 patients and $66 \%$ of G1 patients had motor response to $\mathrm{TI}$, which is not in line with Vernon et al. ${ }^{28}$ who have evidenced that with BIS values below 40 , the likelihood of movement is virtually zero, in anesthetic techniques both with propofol/alfentanil and with isoflurane/alfentanil. It is worth stressing, however, that movement is sub-cortical and BIS directly reflects the cortical component and only indirectly the sub-cortical component ${ }^{29-31}$. Our results suggest dissociation between the levels of hypnosis and analgesia in G1, G2 and G4 since there has been movement at intubation in $\mathrm{G} 1$ and $\mathrm{G} 4$ and a gap above 10 in $\mathrm{G} 2$ (which has also represented $p<0.05$ between moments $M_{2}$ and $M_{3}$ for $R E$ values). Only $\mathrm{G} 3$ has not shown significant RE differences between $M_{2}$ and $M_{3}$, which could have reflected better anesthetic control. In G1, G2 and G3 patients, SBP, DPB and HR have significantly decreased from $M_{1}$ to $M_{3}$, and $M_{3}$ has always shown the lowest scores. Clinically, however, these variations were not relevant. There have been no major changes in G4 in hemodynamic parameters (SBP and DBP) between $M_{1}$ and $M_{3}(p>0.05)$, except for HR $(p<0.05)$ with highest values recorded in $\mathrm{M}_{3}$. There were no motor responses to tracheal intubation maneuvers in $\mathrm{G} 2$ and $\mathrm{G} 3$, which could suggest that $5 \mu \mathrm{g} . \mathrm{kg}^{-1}$ would be the optimal TI dose. Response entropy, however, has shown that $\mathrm{G} 3$ had the most efficient sub-cortical activity block. So, this study has shown that sevoflurane alone, or associated to $2.5 \mu \mathrm{g} . \mathrm{kg}^{-1}$ or $5 \mu \mathrm{g} . \mathrm{kg}^{-1}$ fentanyl has not effectively blocked CNS cortical and sub-cortical components response, as opposed to $7.5 \mu \mathrm{g} . \mathrm{kg}^{-1}$ which has promoted better control of such anesthetic components.

\section{REFERÊNCIAS - REFERENCES}

01. Viertio-Oja HE, Drachman RN, Merilainen PT et al - New method to determine depth of anesthesia from EEG measurements. J Clin Monit Comp, 2000;16:60.

02. Vila P, Canet J, Muñoz S et al - Entropy: a new anesthetic depth monitor. Comparative study with BIS in the clinical setting. Eur J Anaesth, 2003;20:(30):A-91

03. Doi M, Gajraj RJ, Mantzaridis $\mathrm{H}$ et al - Relationship between calculated blood concentration of propofol and electrophysiological variables during emergence from anaesthesia: comparison of bispectral index, spectral edge frequency, median frequency and auditory evoked potential index. Br J Anaesth, 1997;78:180-184.

04. Nishiyama T, Higashizawa T, Bito $\mathrm{H}$ et al - Which laryngoscope is the most stressful in laryngoscopy; Macintosh, Miller, or McCoy? Masui, 1997;46:1519-1524. 
05. Ko SH, Kim DC, Han YJ et al - Small-dose fentanyl: optimal time of injection for blunting the circulatory responses to tracheal intubation. Anesth Analg, 1998;86:658-661.

06. Yli-Hankala A, Vakkauri A, Hoymork S et al - EEG entropy monitoring decreases propofol consumption and shortens early recovery times. Eur J Anesth, 2003;20:(30):A-98.

07. Shannonn CE - A mathematical theory of communication. Bell Syst Tech J, 1948;27:379-423, 623-656.

08. Stein-Ross ML - Theoretical electroencephalogram stationary spectrum for a white-noise-driven cortex: evidence for a general anesthetic-induced phase transition. Phys Rev E Stat Phys Plasmas Fluids Relat Interdiscip Topics, 1999;60:7299-7311.

09. Quiroga RQ, Arnhold J, Lehnertz K et al - Kulback-Leibler and renormalized entropies: applications to electroencephalograms of epilepsy patients, Phys Rev E Stat Phys Plasmas Fluids Relat Interdiscip Topics, 2000;62:8380-8386.

10. Johnson RW, Shore JE - Which is the better entropy expression for speech processing: -S logS or logS? IEEE Acoust Speech Signal Proc, 1984;32:129-137.

11. Lader MH, Mathews AM - Electromyographic studies of tension. J Psychosom Res, 1971;15:479-486.

12. Rosen L, Lunn JN - Consciousness and Awareness and Pain in General Anaesthesia, $1^{\text {st }}$ Ed, London, Butterworths, 1987; 89-98

13. Dement W, Kleitman $\mathrm{N}$ - The relation of eye movements during sleep to dream activity: an objective method for the study of dreaming. J Exp Psychol, 1957;53:339-346.

14. Edmonds HL, Triantafillou T, Tsueda I et al - Comparison of frontalis and hypothenar EMG responses to vecuronium. Anesthesiology, 1985;63:A324.

15. Edmonds HL, Couture LJ, Stolzy SL et al - Quantitative surface electromyography in anesthesia and critical care. Int J Clin Monit Comput, 1986;3:135-145.

16. Edmonds HL, Paloheimo M - Computerized monitoring of the EMG and EEG during anesthesia. An evaluation of the anesthesia and brain activity monitor. Int $\mathrm{J}$ Clin Monit Comput, 1985;1:201-210.

17. Watt RC, Hameroff SR, Cork RC et al - Spontaneous EMG monitoring for anesthetic depth assessment. Proceeding of the Association of Advanced Medical Instrumentation, 1985;20:92.

18. Mathews DM, Kumaran KR, Neuman GG - Bispectral index-derived facial electromyography-guided fentanyl tritation in the opiate-exposed patient. Anesth Analg, 2003;96:1062-1064.

19. Kern SE, David PJ, Dezaire BS et al - Assessing the facial EMG as an indicator of response to noxious stimuli in anesthetized volunteers. ASA Meeting Abstracts, 1999:A594.

20. Shander A, Qin F, Bennett $H$ - Prediction of postoperative analgesic requirements by facial alectromyography during simultaneous BIS monitoring. Eur J Anaesthesiol, 2001;18:(Suppl 21):130.

21. Lennon RL, Hosking MP, Daube JR et al - Effect of partial neuromuscular blockade on intraoperative electromyography in patients undergoing resection of acoustic neuromas. Anesth Analg, 1992:75:729-733.

22. Dutton RC, Smith WD, Bennett $H L$ et al - Craniofacial electromyogram activation response: another indicator of anesthetic depth. J Clin Monit Comput, 1998;14:5-17.
23. Zalunardo MP, Zollinger A, Spahn DR et al - Effects of intravenous and oral clonidine on hemodynamic and plasmacatecholamine response due to endotracheal intubation. J Clin Anesth, 1997;9:143-147.

24. Randell T, Seppala T, Lindgren L - Isoflurane in nitrous oxide and oxygen increases plasma concentrations of noradrenaline but attenuates the pressor response to intubation. Acta Anaesthesiol Scand, 1991;35:600-605.

25. Mi WD, Sakai T, Takahashi S at al - Haemodynamic and electroencephalograph responses to intubationduring induction with propofol or propofol/fentanil. Can J Anaesth, 1998;45:19-22.

26. Billard V, Moulla F, Bourgain JL et al - Haemodynamic response to induction and intubation. Propofol/fentanil interaction. Anesthesiology, 1994;81: 1384-1393.

27. Macleod AD, Maycock E - Awareness during anaesthesia and post traumatic stress disorder. Anaesth Intensive Care, 1992;20:378-382.

28. Vernon JM, Lang E, Sebel PS et al - Prediction of movement using bispectral electroencephalographic analysis during propofol/alfentanil or isoflurane/alfentanil anesthesia. Anesth Analg, 1995;80:780-785.

29. Nunes RR - Componentes da atividade anestésica - uma nova visão. Rev Bras Anestesiol, 2003;53:145-149.

30. Rampil IJ, Mason P, Singh H - Anesthetic potency (MAC) is independent of forebrain structures in the rat. Anesthesiology, 1993;78:707-712.

31. Rampil IJ - Anesthetic potency is not altered after hypothermic spinal cord transection in rats. Anesthesiology, 1994;80:606-610.

\section{RESUMEN}

Nunes RR - Entropía: Un Nuevo Método de Mensuración de la Profundidad de la Anestesia. Estudio Comparativo con el Índice Bispectral en la Evaluación Clínica de la Entubación Traqueal con Sevoflurano

JUSTIFICATIVA Y OBJETIVOS: Entropía espectral, un novo método de análisis del EEG, fundamentado en la cuantificación del caos del EEG, fue desarrollado para monitorización de la profundidad anestésica. Él separa la monitorización en dos tipos de análisis: entropía de estado (SE), que incluye señales de baja frecuencia $(<32 \mathrm{~Hz})$ y entropía de respuesta (RE), que incluye señales con frecuencia hasta $47 \mathrm{~Hz}$. El objetivo de este estudio fue comparar los valores de entropía con los del BIS y respuestas sub-corticales a entubación orotraqueal, en pacientes sometidas a la anestesia general con sevoflurano.

MÉTODO: Participaron del estudio 36 pacientes con edades entre 20 y 44 anos, ASA I, distribuidos en cuatro grupos de nueve, sometidos a la entubación orotraqueal (IOT). En todos los grupos, la anestesia fue inducida con sevoflurano, asociado o no al fentanil, de acuerdo con lo siguiente: (G1 = sevoflurano y 2,5 $\mu \mathrm{g} \cdot \mathrm{kg}^{-1}$ de fentanil; $\mathrm{G} 2$ = sevoflurano y $5 \mu \mathrm{g} \cdot \mathrm{kg}^{-1}$ de fentanil; G3 = sevoflurano y $7,5 \mu \mathrm{g} \cdot \mathrm{kg}^{-1}$ de fentanil y $\mathrm{G} 4=$ sevoflurano $y$ solución fisiológica). Fueron evaluados los siguientes parámetros: PAS, PAD, FC, BIS, SE, RE, concentración expirada del sevoflurano (CE) y respuesta motora a la IOT en tres momentos: $M_{1}=$ inmediatamente antes de la inducción; $M_{2}$ = inmediatamente antes de la entubación traqueal y $M_{3}=$ un minuto después a entubación traqueal.

RESULTADOS: Los valores de BIS y SE variaron de manera linear en todos los grupos, con diferencias significativas entre $M_{2} y$ $M_{3}$ en los grupos $G 1$ y $G 4$, teniendo ambos (BIS y $S E$ ) 
presentando valores arriba de los limítrofes entre consciencia e inconsciencia en el momento $M_{3}$ del G4. En relación al RE, apenas el G3 no mostró variaciones estadísticamente significativas entre los momentos $M_{2}$ y $M_{3}$. Las variaciones hemodinámicas no ultrapasaron valores clínicamente significativos, excepto elevaciones de la FC en el G4 entre los momentos $M_{1}$ y $M_{3}(p<0,05 \%)$. En el $\mathrm{G} 1,66 \%$ de los pacientes reaccionaron a las maniobras de IOT y $100 \%$ en el grupo G4.
CONCLUSIONES: Este estudio indica que el sevoflurano aisladamente, asociado a 2,5 $\mu \mathrm{g} \cdot \mathrm{kg}^{-1}$ o $5 \mu \mathrm{g} \cdot \mathrm{kg}^{-1}$ de fentanil, no bloquea efectivamente las respuestas de los componentes cortical y sub-cortical del SNC, siendo la dosis de 7,5 $\mu \mathrm{g} . \mathrm{kg}^{-1}$ la mejor asociación al sevoflurano para control de estos componentes anestésicos. 